
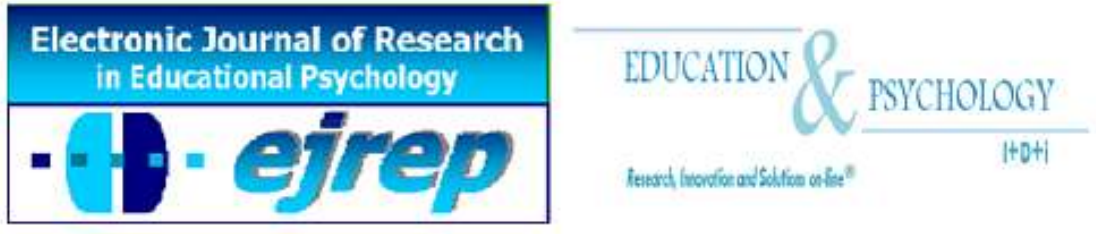

\title{
Conocimiento didáctico sobre números y operaciones: una comparación internacional
}

\section{Araceli Gutiérrez-Gutiérrez ${ }^{1}$, Luis Rico Romero ${ }^{1}$ y Pedro Gómez ${ }^{2}$}

\footnotetext{
${ }^{1}$ Departamento de Didáctica de la Matemática, Universidad de Granada.

${ }^{2}$ Centro de Investigación y Formación en Educación (CIFE) de la Universidad de los Andes, Bogotá.
}

\section{España / Colombia}

Correspondencia: Araceli Gutiérrez-Gutiérrez. Departamento de Didáctica de la Matemática. Facultad de Ciencias de la Educación. Campus de Cartuja. 18071 Granada. España. E-mail: araceligt@correo.ugr.es 


\section{Resumen}

Introducción. En este trabajo se presentan los resultados de comparar el conocimiento didáctico sobre números y operaciones manifestado por los profesores de primaria españoles en formación en el estudio TEDS-M (Teacher Education and Development Study in Mathematics) con el conocimiento manifestado, tanto por los futuros profesores de los países de la OCDE que participaron en el estudio - Noruega, Alemania, Chile, España y Polonia-, como por los futuros profesores de los países participantes receptores de un programa de formación inicial similar al de España — China-Taipéi, Singapur, Estados Unidos, Filipinas y Suiza-.

Método. Para lograr el objetivo propuesto se analizaron los datos y calcularon los parámetros, con base en categorías, que caracterizan el conocimiento didáctico requerido para responder correctamente las preguntas de este dominio conceptual y a partir de criterios de valoración de las respuestas de los futuros profesores.

Resultados. Los futuros maestros presentan, en general, resultados inferiores al del resto de países de la OCDE y al de los países de su grupo de programa de formación. Los resultados españoles son inferiores en todos los aspectos considerados a los resultados de Noruega, Suiza y Singapur. Para la mayoría de las categorías, los resultados españoles son superiores a Filipinas y Chile y próximos a los de EE.UU.

Discusión. Si comparamos los resultados de los países de la OCDE que han participado en los dos estudios internacionales, TEDS-M y TIMSS 2011, —España, Polonia, Estados Unidos, Noruega, Alemania y Chile - podemos comprobar que se conserva la posición relativa de los países en el ranking de rendimientos tanto de profesores en formación como de alumnos de primaria cuando se evalúa su conocimiento matemático sobre números y operaciones. Los resultados españoles en TIMSS son solo superiores a Polonia y Chile e inferiores al resto. Los resultados obtenidos en este trabajo pueden ser de utilidad en el proceso actual de diseño de las asignaturas de Grado de Maestro de Primaria.

Palabras Clave: conocimiento didáctico del contenido, Didáctica de la Matemática, formación inicial de profesores, contenido Números, educación primaria, TEDS-M. 


\title{
Pedagogical knowledge of numbers and operations: an international comparison
}

\begin{abstract}
Introduction. In this paper, we present the results of comparing the mathematics pedagogy knowledge about number and operations that Spanish future primary teachers showed in TEDS-M (Teacher Education and Development Study in Mathematics), first, with the future primary teachers' knowledge of OECD countries that also participated in the study Norway, Germany, Chile y Poland-; and, secondly, with the future primary teachers' knowledge of the participating countries which had followed program-types with similar purposes and characteristics to the Spanish program - China-Taipei, Singapore, United States, Philippines and Switzerland-.

Method. Data analysis will be based on categories that characterize the pedagogical knowledge required to answer correctly the questions in this conceptual domain. We established criteria for assessing the responses of student teachers for calculating the corresponding parameters.

Results. We found that Spanish prospective teachers have lower results, in general, than the other OECD countries and the countries of its group with similar training programs. The Spanish scores are lower than Norwegian, Swiss and Singaporeans scores in all aspects considered. Spain obtained higher scores than Philippines and Chile and next to the U.S.A.

Discussion: Comparing the results of the OECD countries that have participated in two international studies, TEDS-M and TIMSS 2011 — Spain, Poland, USA, Norway, Germany and Chile- we find that the relative position of the country ranking is the same for number and operations. Spanish results in TIMSS are just above Poland and Chile and below the rest. The results obtained in this study may be useful in the current syllabi design process for the Elementary Teacher grade.
\end{abstract}

Keywords: Mathematics Pedagogy, number, pedagogical content knowledge, pre-service teacher education, primary education, TEDS-M.

Reception: 09/05/14 Initial acceptance: 11/09/14 Final acceptance: 02/03/15 


\section{Introducción}

El interés por indagar acerca de la formación inicial en matemáticas de los profesores de primaria en España - maestros, como se les denomina habitualmente - cobra actualidad al producirse la publicación de los informes de evaluaciones internacionales y de los resultados españoles, junto con sus correspondientes estudios secundarios, como son los estudios sobre PISA 2012 (Program for International Student Assessment) (OCDE, 2013; INEE, 2013a; INEE, 2013b), sobre TIMSS 2011(Trends in Mathematics and Science Study) (Martin, M.O. y Mullis, I.V.S., 2013; INEE, 2012a; INEE, 2012b) y TEDS-M 2008 (Teacher Education and Development Study in Mathematics) (Tatto, Sharon, Senk, Ingvarson y Rowley, 2012; INEE, 2012c; INEE, 2012d). Estos estudios coinciden y dan continuidad al interés de las administraciones educativas españolas manifestado en los últimos años con la reforma de los planes de formación inicial de maestros de educación Primaria (ANECA, 2005; MEC, 2007a). Los estudios mencionados muestran una atención sostenida hacia la evaluación educativa, lo que se ha llamado la cultura evaluativa, que implica conocer los puntos fuertes y débiles de la formación matemática de los escolares y de la formación matemática y didáctica de sus profesores en España, así como su comparación con la de otros países.

El objetivo de este trabajo es describir y caracterizar el conocimiento didáctico sobre números y operaciones manifestado por los estudiantes de Magisterio en el estudio TEDS-M y compararlo con el manifestado por los futuros profesores de algunos de los otros países participantes en ese estudio. En concreto, nos centraremos en comparar los resultados españoles con los resultados de aquellos países participantes en TEDS-M que tienen un programa de formación inicial similar al de España, así como con los resultados de los países de la OCDE que también participaron en TEDS-M y que habitualmente son considerados próximos a nuestro entorno socioeconómico y cultural. Para ello, comenzaremos describiendo el estudio TEDS-M y la pertinencia de profundizar en los resultados internacionales del dominio conceptual de números y operaciones. A continuación, describiremos la caracterización y evaluación del conocimiento de la enseñanza de las matemáticas escolares realizada por TEDS-M, con el propósito de describir y caracterizar los conocimientos manifestados por los futuros profesores en el dominio de números y operaciones. Presentaremos los resultados internacionales sobre el conocimiento didáctico y lo interpretaremos, realizando las oportunas compara- 
ciones. Concluiremos mostrando la relevancia de los resultados obtenidos para el diseño actual de las asignaturas del Grado de Maestro de Primaria.

\section{El estudio TEDS-M}

El estudio TEDS-M se llevó a cabo durante los años 2006-2010 y participaron 17 países. La publicación del informe internacional tuvo lugar en el año 2012 (Tatto et al., 2012). Este estudio respondía al interés internacional por la formación inicial de los futuros profesores de matemáticas de educación obligatoria tanto de primaria como de secundaria. Fue patrocinado por la Asociación Internacional para la Evaluación del Rendimiento Educativo (IEA, por sus siglas en inglés) y se basaba en el supuesto de que un factor importante que puede explicar las diferencias en las capacidades, conocimientos y actitudes de los alumnos de primaria y secundaria obligatoria manifestadas en estudios internacionales (como PISA o TIMSS) tiene que ver con la variedad de aproximaciones a la formación inicial del profesorado de matemáticas (Tatto et al., 2012).

España participó en este estudio para, entre otros objetivos, evaluar la formación inicial de los maestros en matemáticas y su didáctica y obtener información que permitiera caracterizar el conocimiento matemático y didáctico con el que los futuros maestros terminaban sus estudios. La coordinación global del estudio TEDS-M en España correspondió a la Secretaría de Estado de Educación y Formación Profesional del Ministerio de Educación a través del Instituto de Evaluación. La coordinación nacional de la investigación estuvo a cargo del profesor Luis Rico, de la Universidad de Granada (INEE, 2012c, p. 131).

Dada la diversidad de programas de formación, y para facilitar las comparaciones internacionales, el equipo de TEDS-M estableció rasgos distintivos con los que identificar los programas de formación inicial de profesores de primaria de los países participantes, dando como resultado una clasificación en cuatro grupos. España quedó incluida en el grupo 2, junto con China-Taipéi, Singapur, Suiza, Estados Unidos y Filipinas, países que coinciden en impartir programas generalistas y preparar a los futuros profesores para dar clase a alumnos de hasta 12 años (INEE, 2012c, p. 24).

Las implicaciones del estudio TEDS-M se manifiestan en los diversos estudios secundarios que se están realizando. Algunos de los aspectos relacionados con la formación inicial de profesores de primaria que abordan estos estudios son los siguientes: la relación entre las 
calificaciones obtenidas en el instituto y los resultados obtenidos en el estudio TEDS-M (Montalvo y Gorgels, 2013); la influencia de las universidades en los resultados de los futuros profesores, mediante un estudio comparativo entre España y Estados Unidos (Cebolla-Boado y Garrido-Medina, 2013); la situación del Prácticum en España en la formación del profesorado (Egido y López, 2013); las creencias de los futuros profesores acerca de la naturaleza de las matemáticas (Felbrich, Kaiser y Schmotz, 2012), etc. También, comienzan a publicarse estudios sobre los conocimientos matemáticos y didácticos manifestados en TEDS-M; estos estudios realizan comparativas internacionales a partir de los resultados. Es el caso, por ejemplo, del estudio de Blömeke, Suhl y Döhrmann (2013), quienes tratan de buscar relaciones entre programas de formación y las preguntas respondidas correctamente. En esta última línea de investigación se encuadra nuestro trabajo.

\section{Elección del dominio conceptual números y operaciones}

La elección del dominio conceptual de números y operaciones para este estudio está motivada por diversas razones. Por una parte, su elección se debe a la importancia de la alfabetización numérica a nivel internacional, que en la actualidad se aprecia en diversas investigaciones (Hardy, 2014). Para el caso español, esta importancia se manifiesta en el currículo español de primaria, donde el bloque de contenidos de números y operaciones forma parte de los tres ciclos de primaria. En la educación primaria se busca alcanzar una eficaz alfabetización numérica, entendida como la capacidad para enfrentarse con éxito a situaciones en las que intervengan los números y sus relaciones, permitiendo obtener información efectiva, directamente o a través de la comparación, la estimación y el cálculo mental o escrito (MEC, 2007b, p. 31555). Los contenidos del bloque de números se contemplan, además, en todas las instituciones españolas de formación inicial de maestros, y sus apartados forman parte del currículo común de esas instituciones (Rico, Gómez y Cañadas, 2014).

Por otra parte, los resultados españoles sobre el conocimiento matemático y didáctico de las matemáticas escolares que presentó el informe internacional de TEDS-M (Tatto et al., 2012, p. 143), al igual que los que presenta el informe nacional (INEE, 2012c, p. 85), son globales. Estos resultados indican únicamente el resultado conjunto obtenido por los futuros profesores españoles en el conocimiento matemático (481) y didáctico del contenido (492) sobre una media internacional de 500. Estas razones y argumentos incrementan la necesidad de describir y caracterizar el conocimiento que tienen los futuros profesores de primaria españoles sobre números y su enseñanza, y ubicarlo a nivel internacional. 


\section{Conocimiento didáctico sobre números y operaciones}

El estudio TEDS-M, siguiendo las ideas de Shulman (1987), consideró que el conocimiento matemático para la enseñanza tiene dos componentes —el conocimiento del contenido matemático y el conocimiento didáctico del contenido matemático- y diseñó un cuestionario que evaluaba por separado el dominio de los profesores en formación sobre ambos tipos de conocimientos. Al mismo tiempo, basándose en el marco conceptual de TIMSS 2007, TEDSM organizó las preguntas según cuatro dominios conceptuales: números y operaciones, geometría y medida, álgebra y funciones, y datos y azar (Mullis, Martin, Ruddock, O’Sullivan, Arora, y Erberber, 2007).

El estudio TEDS-M también evaluó el conocimiento didáctico sobre matemáticas escolares de los futuros profesores de primaria con base en 22 preguntas que interrogan sobre cómo abordar diversas tareas y problemas matemáticos escolares. Algunas de las preguntas del cuestionario provenían de estudios como Learning Mathematics for Teaching Projects (Hill y Ball, 2004) y Mathematics Teaching for the 21 st Century Project (Schmidt, Blömeke y Tatto, 2011). El resto de las preguntas fueron elaboradas por el equipo de TEDS-M.

Este estudio se centra en las 8 preguntas que evalúan el conocimiento didáctico del contenido matemático de números y operaciones. En trabajos previos, concluimos que "TEDS-M no presenta una estructura de categorías propia y completa que permita establecer el conocimiento didáctico del contenido matemático de cada pregunta" (Gutiérrez-Gutiérrez, Gómez y Rico, 2014, p. 283), por lo que seleccionamos un conjunto de categorías con las que caracterizamos el conocimiento didáctico requerido para responder correctamente las preguntas de este dominio conceptual. Las categorías, que se presentan a continuación, surgen del análisis de los enunciados de las preguntas y de sus guías de corrección y derivan de las establecidas en el método del análisis didáctico (Rico, 2013).

- Identificar y distinguir variables que afectan a la dificultad de un problema (DIF).

- Reconocer y describir los errores en los que incurren los alumnos al realizar una actividad o sus concepciones erróneas sobre un concepto o procedimiento determinado (RE).

- Representar alternativamente conceptos y procedimientos matemáticos en el proceso de enseñanza (REP). 
- Reconocer aquellos conceptos y procedimientos matemáticos involucrados en la enseñanza de un tema de las matemáticas escolares y las relaciones entre ellos (CPM).

Las dos primeras categorías se refieren al conocimiento que el futuro profesor puede manifestar sobre limitaciones en el aprendizaje de los escolares - las dificultades relacionadas con el conocimiento matemático o los errores en que pueden incurrir al abordar tareas matemáticas - Las otras dos categorías hacen referencia al conocimiento de un contenido matemático escolar y aluden a sus representaciones y a la estructura conceptual de ese contenido

\section{Objetivo}

Como se ha dicho, el objetivo de esta investigación es describir y caracterizar el conocimiento didáctico sobre números y operaciones manifestado por los futuros profesores de primaria españoles que participaron en el estudio TEDS-M y compararlo por una parte con el conocimiento manifestado por los futuros profesores de los países de la OCDE que intervinieron en el estudio - Noruega, Alemania, Chile y Polonia - y, por otra, con el manifestado por los futuros profesores de los países participantes pertenecientes al mismo grupo que España -China-Taipéi, Singapur, Estados Unidos, Filipinas y Suiza - denominado grupo 2. Se trata, por tanto, de una investigación comparativa, a partir de los datos procedentes del cuestionario del estudio TEDS-M.

\section{Método}

\section{Participantes}

El trabajo de campo siguió el diseño establecido por TEDS-M (Tatto, Schwille, Senk, Ingvarson, Peck y Rowley, 2008). En cada país, se seleccionaron muestras representativas de las instituciones que ofrecían formación a la población diana de futuros profesores que se estaban preparando para enseñar matemáticas en primaria y que se encontraban en su último año de formación. Esta selección se hizo aplicando un muestreo con probabilidad proporcional al tamaño de la institución, definido tal tamaño en términos del número de estudiantes para profesor de primaria que cursaban último curso en el año 2008. En cada institución, se seleccionó una muestra aleatoria de 30 futuros profesores - o la población completa si su tamaño era inferior a 30-. Participaron un total de 483 instituciones con sus respectivos pro- 
gramas de formación y 13871 futuros profesores de primaria de matemáticas de dichas instituciones (Tatto et al., 2012).

La muestra de instituciones españolas estuvo compuesta por 50 de un total de 73 instituciones que ofrecían formación inicial a futuros maestros de primaria. Dos de ellas declinaron la invitación a participar. Un total de 1093 futuros maestros españoles, que seguían el programa de formación establecido por el Real Decreto 1440/1991 (MEC, 1991) anterior al actual título de Grado, respondieron el cuestionario (INEE, 2012c).

\section{Instrumentos}

Este estudio se basa en la información proporcionada por el cuestionario de TEDS-M, las guías de corrección diseñadas para la corrección de las preguntas de respuesta abierta y las respuestas de los futuros profesores participantes a dicho cuestionario.

\section{Procedimiento}

Para lograr el objetivo propuesto, seguimos un procedimiento compuesto de tres pasos:

- selección, análisis y clasificación de las preguntas del dominio de números y operaciones;

- establecimiento de criterios de valoración de las respuestas de los futuros profesores y cálculo de parámetros;

- resumen de los datos en términos de medias y comparación de dichas medias.

1) Selección, análisis y clasificación de las preguntas del dominio de números y ope raciones

En trabajos previos, presentamos el proceso de identificación, análisis y clasificación de las preguntas (Gutiérrez, 2012; Gutiérrez-Gutiérrez, Gómez y Rico, 2014). El resultado de ese proceso se muestra en la tabla 1 que incluye las 8 preguntas con las que se evaluó el conocimiento didáctico del contenido para números y operaciones en el estudio TEDS-M. En ella indicamos, para cada pregunta, su contenido matemático concreto, el conocimiento didáctico específico que se requiere para contestarla correctamente -identificado por las categorías establecidas - y el tipo de respuesta. Como es habitual en este tipo de estudios, las preguntas del cuestionario son de respuesta múltiple — en el caso del dominio de números y operaciones se proporcionaban en cada pregunta 4 opciones de respuesta de la cuales solo una era correc- 
ta—; de respuesta múltiple compuesta — cada opción de respuesta del problema (4) se presenta como un apartado con otras dos opciones de respuesta-; o de respuesta abierta. Para estas últimas, el estudio TEDS-M diseñó las guías de corrección correspondientes y su procedimiento de codificación.

Tabla 1. Preguntas de conocimiento didáctico sobre números y operaciones

\begin{tabular}{clcc}
\hline $\begin{array}{c}\text { Número } \\
\text { de } \\
\text { pregunta }\end{array}$ & \multicolumn{1}{c}{ Contenido matemático } & $\begin{array}{c}\text { Conocimiento } \\
\text { didáctico }\end{array}$ & $\begin{array}{c}\text { Tipo de Res- } \\
\text { puesta }\end{array}$ \\
\hline 1 & Problemas aritméticos & DIF & Abierta \\
2 & Proporcionalidad directa entre magnitudes & DIF & Abierta \\
3 & Operaciones con números decimales & RE & Abierta \\
4 & Representación de números decimales & REP & Abierta \\
5 & Orden de fracciones & CPM & Múltiple \\
6 & Interpretación gráfica de la división de & REP & Múltiple com- \\
puesta \\
7 & Oracciones & RE & Múltiple \\
8 & Operaciones con números mixtos & CPM & Múltiple \\
\hline Fuente: Gutiérrez-Gutiérrez, Gómez y Rico (2014, p. 284) & &
\end{tabular}

Fuente: Gutiérrez-Gutiérrez, Gómez y Rico (2014, p. 284)

Mostramos a continuación, en la figura 1, un ejemplo de ítem liberado del cuestionario TEDS-M sobre el conocimiento didáctico de las operaciones con números decimales. Dicho ítem se compone de dos apartados que se clasifican y corrigen de forma independiente. Según la clasificación que hemos hecho de las preguntas, el apartado (a) lo identificamos como la pregunta 3 y el apartado (b) como la pregunta 4.

[Jeremy] se da cuenta de que cuando introduce $0,2 \times 6$ en la calculadora el resultado es menor que 6 , y que cuando introduce $6: 0,2$ tiene un resultado mayor que 6 . Él está perplejo por esto, y le pide a su profesor ¡una nueva calculadora!

a) ¿Cuál es la concepción errónea más probable [de Jeremy]?

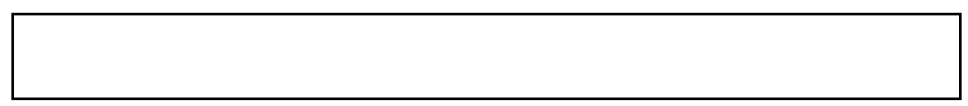

b) Haz una representación gráfica que el profesor podría usar como modelo para representar 0,2 x 6 y ayudar a Jeremy a entender la respuesta a la pregunta 3.

Figura 1. Ítem sobre números decimales 
Sucintamente presentaremos a continuación los conocimientos que debían manifestar los futuros profesores para contestar correctamente cada uno de los apartados según las guías de corrección. Propondremos algunos ejemplos donde se muestre la presencia del conocimiento evaluado en la literatura de formación inicial de profesores, singularmente mediante algunos documentos españoles. Por último, sintetizaremos el conocimiento didáctico del contenido matemático evaluado mediante este ítem.

El apartado (a) evalúa el conocimiento de los futuros profesores de primaria sobre las limitaciones en el aprendizaje de los escolares. La guía de corrección establece que, para contestarlo correctamente, basta que los futuros profesores, en sus respuestas, sugieran que la concepción errónea del alumno sea considerar que la multiplicación de números decimales siempre da un resultado mayor que los números propuestos, y que la división siempre da un resultado menor que el dividendo. La literatura sobre errores en los que incurren los escolares al operar con números decimales es amplia. Por ejemplo, Castro (2001) recoge la concepción errónea que se evalúa en este problema y que se refiere a que los niños consideren que "multiplicar es siempre aumentar y dividir es siempre disminuir. Esta concepción equivocada la mantienen los niños con números decimales positivos menores que la unidad” (p. 335).

El apartado (b) evalúa un aspecto del conocimiento didáctico de los futuros profesores sobre el contenido matemático. Se pide al futuro profesor que, de manera gráfica, represente el producto de 0,2 x 6 de tal forma que el alumno pueda comprender el error conceptual sobre la multiplicación con números decimales en el que ha incurrido en el apartado anterior. Es decir, se evalúa el conocimiento del futuro profesor sobre la representación gráfica de los números decimales en general y de la multiplicación de un número decimal entre 0 y 1 por un número natural en particular. La guía de corrección establece que para contestar correctamente a este apartado el futuro profesor debe saber que "los números decimales proporcionan una ampliación del sistema decimal de numeración: con los números naturales se representan cantidades enteras; con los números decimales se expresan también las diferentes partes de la unidad" (Castro, 2001, p. 320). Mostramos en la figura 2 dos representaciones que se proponen como ejemplos de respuestas correctas tal y como aparecen en la guía de corrección. En estas guías de corrección no se contempla el uso implícito de la propiedad conmutativa del producto, cuando las representaciones mostradas corresponden al producto 6 x 0,2 y no al producto $0,2 \times 6$. 


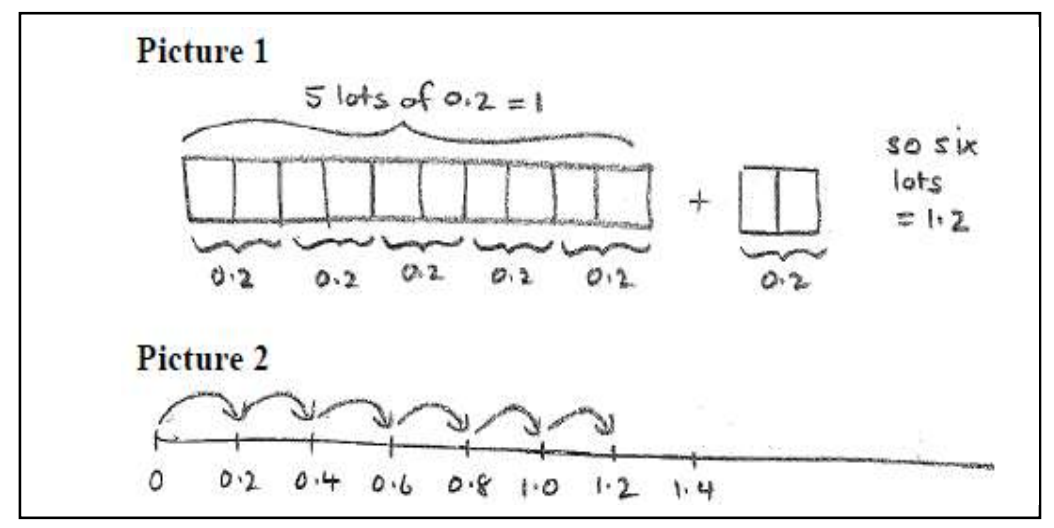

Figura 2. Ejemplos de respuestas correctas del apartado (b)

Fuente: Imagen original de la guía de corrección diseñada por TEDS-M

En la primera de las figuras (Picture 1) el futuro profesor manifiesta reconocer la representación gráfica de los números decimales como sub-áreas de una región que se toma como unidad. En el caso del ejemplo, 0,2 se representa como 2/10. En la segunda figura (Picture 2), el futuro profesor considera los decimales como puntos sobre la recta numérica. En ambas representaciones se identifica que 0,2 x 5 corresponde a la unidad.

Ante esto, concluimos que para que un futuro profesor de primaria conteste correctamente el ítem propuesto en la figura 1 no le basta tener un conocimiento matemático de los números decimales y de las operaciones con ellos. Es además necesario que disponga de un conocimiento didáctico sobre la enseñanza de los números decimales, su representación gráfica, los errores más frecuentes en los que pueden incurrir los alumnos y la aplicación de las representaciones gráficas en la enseñanza. Los apartados (a) y (b) dan información del conocimiento didáctico de los futuros profesores sobre los números decimales, pero cada uno proporciona información sobre un aspecto concreto de ese conocimiento didáctico.

2) Establecimiento de criterios de valoración de las respuestas de los futuros profesores

Las respuestas de los participantes en el estudio fueron codificadas según la guía de puntuación desarrollada por TEDS-M (Tatto et al., 2008). El sistema de puntuación para cada pregunta de respuesta abierta es un código de dos dígitos. El primer dígito, un 2 o un 1, indica una respuesta correcta o parcialmente correcta respectivamente y también significa el valor con el que se califica la respuesta. Mientras que las respuestas incorrectas se identifican con 
un 7 como primer dígito. El segundo dígito se refiere a diferentes enfoques utilizados por los futuros profesores en sus respuestas. En nuestro caso y de forma similar a como se hace en TEDS-M para calcular los resultados generales (Tatto et al., 2008, p. 42), valoramos las respuestas de los futuros profesores a cada pregunta atendiendo a los distintos tipos de respuesta.

- Si la pregunta es de respuesta múltiple asignamos el valor 1 si el futuro profesor contestó correctamente y 0 si contestó de forma incorrecta.

- Si la pregunta es de respuesta abierta asignamos el valor 1 si el futuro profesor contestó correctamente, 0 si contestó de forma incorrecta y 0,5 si contestó de forma parcialmente correcta. Consideramos en el último caso, a partir del análisis de las guías de corrección que realizaron Gutiérrez (2012) y Gutiérrez-Gutiérrez, Rico y Gómez (2014), que el conocimiento manifestado en los distintos tipos de respuestas parcialmente correctas merece la misma puntuación.

- Si la pregunta es de respuesta múltiple compuesta asignamos el valor 1 si el futuro profesor contesta correctamente todas las opciones de respuesta, 0,5 si contesta correctamente todas menos una, y 0 para el resto de los casos. En Tatto et al. (2008, p. 76) se puede consultar un ejemplo del análisis de valoración para la corrección de una pregunta de este tipo.

- Las respuestas ilegibles, tachadas o en blanco se consideran valores perdidos.

\section{3) Resumen de los datos}

Los parámetros que utilizamos para realizar las comparaciones con respecto al conjunto de preguntas que evaluaban el conocimiento didáctico de números y a cada una de las categorías del conocimiento didáctico establecidas, fueron los siguientes.

- La media y la desviación típica de cada uno de los países.

- La media y la desviación típica del total de países participantes en TEDS-M.

- La media y la desviación típica del conjunto de países participantes que forman parte de la OCDE.

- La media y la desviación típica del conjunto de países que forman parte del grupo 2.

Hacemos la presentación de estos parámetros mediante dos tablas. En la tabla 2 presentamos la media y la desviación típica de España — para el total de preguntas que evaluaban el conocimiento didáctico de números y operaciones (CD), y para cada una de las categorías establecidas - con respecto a la media y la desviación típica del conjunto de países partici- 
pantes y la media así como a la desviación típica del conjunto de países pertenecientes a la OCDE —incluido España - que participaron en TEDS-M. En la tabla 3 presentamos la misma información española e internacional pero en este caso con respecto a la media y desviación típica de los países del grupo 2. En ambas señalamos los países que tienen resultados superiores e inferiores a España para el conjunto de preguntas consideradas y para cada una de las categorías del conocimiento didáctico establecidas. No hemos incluido en la tabla los países cuyas medias no son estadísticamente superiores o inferiores a la de España con un nivel de significación del 0,05 en cada caso considerado. En el caso de países con más de un programa de formación, identificamos el programa específico.

Presentamos en el anexo del documento, como ejemplo, las figuras 3, 4 y 5 en las que mostramos la media y la desviación típica de cada uno de los países participantes para el conjunto de preguntas que evaluaban el conocimiento didáctico del dominio de números, así como para la categoría DIF y la categoría RE. En estas figuras, para cada país, se representa su correspondiente intervalo de confianza mediante un segmento dentro del cual se sitúa la puntuación media real con una confianza del 95 por ciento; la mayor o menor amplitud del intervalo depende del tamaño de la muestra y de la dispersión de los resultados. Y en cada una de las figuras, se identifica, mediante una franja roja, la media internacional y una desviación típica alrededor de ella.

Estos y los demás resultados que aparecen en este trabajo deben leerse teniendo en cuenta las limitaciones de participación de Chile, Estados Unidos, Noruega, Rusia, Polonia y Suiza (INEE, 2012c, p.136).

Tabla 2. Resumen comparativo de los resultados de España con los países de la OCDE

\begin{tabular}{|c|c|c|c|c|c|c|c|c|}
\hline \multirow[t]{2}{*}{ CAT } & \multicolumn{2}{|c|}{ ESPAÑA } & \multicolumn{2}{|c|}{ Internacional } & \multicolumn{4}{|c|}{ PAÍSES DE LA OCDE } \\
\hline & $\mathrm{Me}$ & $\mathrm{E}$ & $\mathrm{Me}$ & $\mathrm{E}$ & $\mathrm{Me}$ & $\mathrm{E}$ & Me Sup & Me Inf \\
\hline $\mathrm{CD}$ & 0,39 & 0,01 & 0,44 & 0,00 & 0,43 & 0,00 & $\begin{array}{c}\text { Noruega } \\
\text { Suiza } \\
\text { Alemania } \\
\text { Polonia Esp } \\
\text { Noruega } \\
\text { Suiza }\end{array}$ & Polonia Gen \\
\hline DIF & 0,70 & 0,02 & 0,72 & 0,01 & 0,63 & 0,00 & $\begin{array}{c}\text { Alemania } \\
\text { EE.UU. } \\
\text { Polonia Gen }\end{array}$ & Polonia Esp \\
\hline $\mathrm{RE}$ & 0,23 & 0,02 & 0,31 & 0,01 & 0,37 & 0,01 & $\begin{array}{c}\text { Polonia } \\
\text { Noruega } \\
\text { Alemania }\end{array}$ & Chile \\
\hline
\end{tabular}




\begin{tabular}{|c|c|c|c|c|c|c|c|c|}
\hline \multirow[t]{3}{*}{ CAT } & \multicolumn{2}{|c|}{ ESPAÑA } & \multicolumn{2}{|c|}{ Internacional } & \multicolumn{4}{|c|}{ PAÍSES DE LA OCDE } \\
\hline & $\mathrm{Me}$ & E & $\mathrm{Me}$ & E & $\mathrm{Me}$ & E & Me Sup & Me Inf \\
\hline & & & & & & & $\begin{array}{c}\text { Suiza } \\
\text { Alemania } \\
\text { Suiza }\end{array}$ & \\
\hline REP & 0,24 & 0,02 & 0,30 & 0,01 & 0,31 & 0,01 & $\begin{array}{c}\text { Noruega } \\
\text { Polonia }\end{array}$ & Chile \\
\hline CPM & 0,33 & 0,01 & 0,36 & 0,01 & 0,35 & 0,01 & $\begin{array}{l}\text { EE.UU. Gen } \\
\text { Noruega+ } \\
\text { Suiza } \\
\text { Polonia Esp }\end{array}$ & $\begin{array}{l}\text { Polonia Gen } \\
\text { EE.UU. Gen } \\
\text { Chile }\end{array}$ \\
\hline
\end{tabular}

Nota: $\mathrm{CAT}=$ categorías; $\mathrm{CD}=$ conocimiento didáctico de números y operaciones; $\mathrm{DIF}$ = distinguir variables que afectan a la dificultad de un problema; $\mathrm{RE}=$ reconocer los errores en los que incurren los alumnos al realizar una actividad; REP = representar alternativamente conceptos y procedimientos matemáticos en el proceso de enseñanza; $\mathrm{CPM=}$ reconocer los conceptos y procedimientos matemáticos involucrados en la enseñanza de un tema de las matemáticas escolares y las relaciones entre ellos; Me: media; E: error típico; Inf = inferior; Sup = superior; Noruega+ = programa noruego con opción de matemáticas; Gen= programa generalista; Esp= programa especialista.

Tabla 3. Resumen comparativo de los resultados de España con los países del grupo 2

\begin{tabular}{|c|c|c|c|c|c|c|c|c|}
\hline \multirow[t]{2}{*}{ CAT } & \multicolumn{2}{|c|}{ ESPAÑA } & \multicolumn{2}{|c|}{ INTERNACIONAL } & \multicolumn{4}{|c|}{ GRUPO 2} \\
\hline & $\mathrm{Me}$ & E & $\mathrm{Me}$ & E & $\mathrm{Me}$ & E & Me Sup & Me Inf \\
\hline $\mathrm{CD}$ & 0,39 & 0,01 & 0,44 & 0,00 & 0,44 & 0,01 & $\begin{array}{l}\text { China-Taipéi } \\
\text { Singapur } \\
\text { Suiza }\end{array}$ & Filipinas \\
\hline DIF & 0,70 & 0,02 & 0,72 & 0,01 & 0,69 & 0,01 & $\begin{array}{l}\text { Singapur } \\
\text { EE.UU. } \\
\text { Suiza }\end{array}$ & $\begin{array}{l}\text { China-Taipéi } \\
\text { Filipinas }\end{array}$ \\
\hline RE & 0,23 & 0,02 & 0,31 & 0,01 & 0,32 & 0,01 & $\begin{array}{c}\text { Singapur } \\
\text { China-Taipéi } \\
\text { Suiza }\end{array}$ & Filipinas \\
\hline REP & 0,24 & 0,02 & 0,30 & 0,01 & 0,33 & 0,01 & $\begin{array}{c}\text { China-Taipéi } \\
\text { Singapur } \\
\text { Suiza } \\
\text { EE.UU. }\end{array}$ & Filipinas \\
\hline CPM & 0,33 & 0,01 & 0,36 & 0,01 & 0,39 & 0,01 & $\begin{array}{c}\text { China-Taipéi } \\
\text { Suiza } \\
\text { Singapur }\end{array}$ & $\begin{array}{l}\text { EE.UU. } \\
\text { Filipinas }\end{array}$ \\
\hline
\end{tabular}

Nota: $\mathrm{CAT}$ = categorías; $\mathrm{CD}$ = conocimiento didáctico de números y operaciones; $\mathrm{DIF}=$ distinguir variables que afectan a la dificultad de un problema; $\mathrm{RE}=$ reconocer los errores en los que incurren los alumnos al realizar una actividad; REP = representar alternativamente conceptos y procedimientos matemáticos en el proceso de enseñanza; $\mathrm{CPM}$ = reconocer los conceptos y procedimientos matemáticos involucrados en la enseñanza de un tema de las matemáticas escolares y las relaciones entre ellos; Me: media; E: error típico; Inf = inferior; Sup $=$ superior.

Pasamos a continuación a señalar los aspectos más sobresalientes que surgen de interpretar la información contenida en las tablas 2 y 3 y en figuras como las ejemplificadas en los anexos. 


\section{Resultados}

La información que nos proporcionan las tablas y figuras nos permite determinar los siguientes resultados.

\section{Resultados españoles con respecto a los países de la OCDE}

La media española es superior a la media de los países de la OCDE solo para la categoría DIF. Para el resto de categorías es siempre inferior. España obtiene resultados inferiores a Noruega y Suiza en todos los aspectos considerados. Con respecto a Alemania, con sus dos programas, obtiene también resultados inferiores excepto para la categoría CPM. Polonia, con su programa especialista, presenta resultados superiores a los españoles para todas las categorías. La media polaca con el programa generalista es inferior a la española para el conjunto de preguntas que evaluaban el dominio de números excepto para la categoría CPM. Los resultados españoles son superiores a Chile para todas las categorías excepto para la categoría DIF y $\mathrm{CD}$ donde no hay diferencias significativas. El país cuyos resultados están más próximos a los españoles es EE.UU. con sus dos programas.

Los mejores resultados del grupo de países de la OCDE para el conjunto de preguntas del dominio de números los obtienen Noruega (ALU+) $(0,59)$ y Alemania y Polonia $(0,58)$. La diferencia de la media española con ellas es de 0,20 y 0,19 respetivamente. La diferencia de puntos entre los países de la OCDE con mayor y menor puntuación es 0,22 para el conjunto de preguntas del dominio de números y operaciones. Destaca la escasa variabilidad de los resultados españoles para cada uno de los aspectos estudiados en este trabajo. Los países de la OCDE que presentan una gran dispersión en los resultados son los programas especialistas de Alemania y EE.UU. y el generalista de Polonia. Esta variabilidad está relacionada con el tamaño de las muestras respectivas que oscilan entre 85 y 135 para los casos mencionados, mientras que la española está constituida por 1093 futuros profesores.

\section{Resultados españoles con respecto a los países del grupo 2}

La media española es inferior a la media de los países del grupo 2 excepto para la categoría DIF con la que no hay diferencia significativa. Los resultados españoles son inferiores en todos los aspectos considerados a los resultados de Suiza y Singapur. Destaca la media española en la categoría DIF que supera ampliamente la media de China-Taipéi, siendo en el resto de categorías siempre inferior. Los resultados españoles son superiores a Filipinas para 
todas las categorías. Con respecto a EE.UU., no hay diferencias significativas con excepción de la categoría CPM donde la media española supera a la estadounidense. Los resultados de los países del grupo 2 presentan, en general, una escasa variabilidad. Solo Singapur y Filipinas superan en escasas ocasiones la desviación típica de 0,02 mientras que la española es siempre menor o igual que dicho valor.

A nivel del conjunto de países del grupo 2, Singapur y Suiza son los países que obtienen los mejores resultados para todas las categorías. China-Taipéi también se encuentra entre ellos excepto para la categoría DIF. Para el conjunto de preguntas que componen el dominio de números y operaciones la máxima puntuación la obtiene China-Taipéi $(0,55)$ y España se diferencia con ella en 0,16 .

\section{Resultados españoles con respecto al conjunto de países participantes}

Para el conjunto de categorías, la media de los resultados españoles, siendo siempre inferior, está más próxima a la media internacional del conjunto de países participantes que a la media del grupo 2, con excepción de la categoría DIF donde España obtiene sus mejores resultados. España obtiene los resultados más bajos para las categorías RE y REP, y lo mismo ocurre para el conjunto de países del grupo 2 y para el total de países participantes. Mientras que para los países de la OCDE los resultados más bajos los obtienen en las categorías REP y CPM. El país que obtiene la media más alta es Singapur con su programa especialista, la diferencia con respecto a la media española es 0,21 .

Dada la calidad de la muestra española, los resultados españoles presentan escasa variabilidad para el conjunto de aspectos considerados. No obstante países cuya muestra era inferior a 140, como es el caso de Alemania y EE.UU. con su programa especialista, o Botsuana, Suiza y Noruega (ALU+) con su programa generalista, presentan una gran variabilidad.

\section{Discusión y conclusiones}

Este trabajo contribuye a profundizar en el conocimiento didáctico de los futuros profesores españoles sobre números y operaciones con respecto al resto de países participantes en el estudio. Los resultados presentados identifican deficiencias en su formación que están en consonancia con el papel que tenía la Didáctica de la Matemática en los programas de forma- 
ción inicial de maestros del periodo 1991-2010, donde "España se situaba en las posiciones inferiores con respecto al resto de países, tanto a nivel global como con respecto a los de su grupo en cuanto a la proporción de temas estudiados de Didáctica de la Matemática" (Rico, Gómez y Cañadas, 2014). La comunidad de profesores y expertos de esta disciplina ya percibía que la formación era claramente insuficiente para ejercer como maestro y tener la responsabilidad de la formación matemática de los niños de Educación Primaria. Hay "una contradicción profunda entre la importancia concedida a la competencia matemática de los ciudadanos y la formación de los profesionales encargados de la educación matemática en los decisivos primeros niveles escolares" (Rico, Sierra y Castro, 2002, p. 43).

El interés por el análisis de los resultados obtenidos por los futuros profesores españoles viene avalado por la calidad de la muestra española (INEE, 2012C, p. 17). No obstante a la hora de realizar comparaciones internacionales deben tenerse en cuenta las limitaciones de participación de Chile, Estados Unidos, Noruega, Rusia, Polonia y Suiza (INEE, 2012c, p. 136).

España obtiene la media más baja $(0,23)$ para la categoría de reconocer el error en el que incurre un alumno. Destacamos este hecho para la formación inicial de maestros, dado que la LOMCE prevé la detección precoz de dificultades en el aprendizaje como objetivo, dentro de la atención personalizada (MECD, 2013b). Al mismo tiempo señalamos que el conocimiento sobre errores evaluado en el cuestionario de TEDS-M se contempla en los manuales de formación de profesores de primaria españoles como el de Castro (2001). España obtiene también una media baja $(0,24)$ para la categoría de representar alternativamente conceptos y procedimientos matemáticos en el proceso de enseñanza, este dato también tiene interés pues "las representaciones juegan un papel fundamental en el pensamiento matemático, favorecen la comprensión de los conceptos matemáticos y estimulan el desarrollo de un pensamiento flexible y versátil en la resolución de problemas" (Villegas, Castro y Gutiérrez, 2009, p. 280).

Hemos visto que el cuestionario de TEDS-M contiene preguntas en la que se constata que el conocimiento matemático que puede tener un futuro profesor no basta para reconocer los errores en los que puede incurrir un alumno o para conocer las representaciones gráficas en la enseñanza de un tema determinado. Este hecho destaca la necesidad de incidir no solo en el conocimiento matemático del maestro en formación sino también en el conocimiento de la 
enseñanza-aprendizaje de las matemáticas escolares. Por otra parte, si comparamos los resultados de los países de la OCDE que han participado en los dos estudios internacionales, TEDS-M y TIMSS 2011, —España, Polonia, Estados Unidos, Noruega, Alemania y Chilepodemos comprobar que se conserva la posición relativa de los países en el ranking de rendimientos tanto de profesores en formación como de alumnos de primaria cuando se evalúa su conocimiento matemático sobre números y operaciones. Los resultados españoles en TIMSS son solo superiores a Polonia y Chile e inferiores a los del resto (INEE, 2013a, p. 62).

Aunque no formaba parte del objetivo de este trabajo, hemos encontrado que los mejores resultados se obtienen para todos los países, con diferencia, en aquellas preguntas que evaluaban acerca del conocimiento de los futuros profesores sobre las variables que afectan a la dificultad de los problemas. Como podemos comprobar en las tablas y figuras de los anexos, China-Taipéi, Noruega (ALU+), y los países con programas de formación de especialistas Alemania, Polonia y Singapur — con sus dos programas - presentan, con algunas excepciones, los mejores resultados en las cuatro categorías de conocimiento didáctico establecidas (también las mayores dispersiones). Al mismo tiempo si nos fijamos en las tablas 2 y 3 y en las gráficas de las figuras de los anexos encontramos que hay una gran variabilidad en los resultados en función no solo de los programas sino también del tipo de contenido matemático y de la categoría de conocimiento didáctico que se evalúa en las preguntas.

Las medidas que están tomando los diferentes países para mejorar la aptitud profesional de los profesores, como es el caso de Finlandia, Polonia o Singapur, ponen de manifiesto una preocupación compartida por ampliar y mejorar el nivel de estudios requerido para ser profesor. Éste debería ser el objetivo general de las reformas emprendidas en España: "mejorar la formación inicial que reciben nuestros profesores para responder a las nuevas demandas de una sociedad en cambio" (Esteve, 2006, p. 19). Aunque el estudio es parcial por razón del dominio considerado y de las categorías que se pueden identificar en las preguntas, las categorías estudiadas son transversales a todos los dominios (Gómez y Gutiérrez-Gutiérrez, 2014). Por tanto, los resultados obtenidos en este trabajo pueden ser de utilidad en el proceso actual de diseño y puesta en práctica de las asignaturas de Grado de Maestro de Primaria. 


\section{Agradecimientos}

Este trabajo ha sido apoyado por el Proyecto de Excelencia de la Junta de Andalucía P07-FQM03244 “TEDS-M España” y parcialmente subvencionado por el proyecto EDU2009-10454 del Ministerio de Ciencia e Innovación. También se ha realizado en el marco del proyecto Procesos de aprendizaje del profesor de matemáticas en formación, EDU2012-33030 del MEC. Los resultados de este trabajo forman parte de la tesis doctoral que lleva a cabo A. Gutiérrez-Gutiérrez, bajo la dirección de los doctores L. Rico y P. Gómez.

\section{Referencias}

ANECA (2005). Libro blanco. Título de Grado en Magisterio (volumen 1 y 2). Madrid: Agencia Nacional de Evaluación de la Calidad y Acreditación.

Blömeke, S., Suhl, U. y Döhrmann, M. (2013). Assessing strengths and weaknesses of teacher knowledge in asia, eastern europe, and western countries: differential item functioning in TEDS-M. International Journal of Science and Mathematics Education 11(4), 795 817.

Castro, E. (2001). Números decimales. En E. Castro (Ed.) Didáctica de la Matemática en la educación primaria (pp. 315-345). Madrid: Síntesis.

Cebolla-Boado, H. y Garrido-Medina, L. (2013). Los efectos de la educación universitaria en el conocimiento en matemáticas en España y en EE.UU.: evidencias del cuestionario TEDS-M. En INEE (Ed.), TEDS-M. Estudio internacional sobre la formación inicial en matemáticas de los maestros. Informe español. Volumen II. Análisis secundario (pp. 41-59). Madrid: Autor.

Egido, I. y López, E. (2013). Análisis del Prácticum en los estudios de magisterio en españa a partir de los datos de TEDS-M. En INEE (Ed.), TEDS-M. Estudio internacional sobre la formación inicial en matemáticas de los maestros. Informe español. Volumen II. Análisis secundario (pp. 108-135). Madrid: Autor.

Esteve, J. M. (2006). La profesión docente en Europa: perfil, tendencias y problemática. La formación inicial. Revista de Educación, 340, 19-86.

Felbrich, A., Kaiser, G., y Schmotz, C. (2012). The cultural dimension of beliefs: An investigation of future primary teachers' epistemological beliefs concerning the nature of 
mathematics in 15 countries. The International Journal on Mathematics Education, 44(3), 355-366.

Gómez, P. y Gutiérrez, A. (2014). Conocimiento matemático y conocimiento didáctico del futuro profesor español de primaria. Resultados del estudio TEDS-M. En M. T. González, M. Codes, D. Arnau y T. Ortega (Eds.), Investigación en Educación Matemática XVIII (pp. 83-92). Salamanca: SEIEM

Gutiérrez, A. (2012). Conocimiento didáctico sobre números y operaciones de los estudiantes españoles de Magisterio en TEDS-M. Trabajo Fin de Máster. Universidad de Granada. Disponible en: http://funes.uniandes.edu.co/1921/

Gutiérrez-Gutiérrez, A., Gómez, P. y Rico, L. (2014). Conocimiento didáctico de los estudiantes españoles de Magisterio sobre números: resultados en TEDS-M. Cultura y Educación, 26(2), 265-297.

Gutiérrez-Gutiérrez, A., Rico, L., y Gómez, P. (2014). Metodología para una comparación internacional del conocimiento didáctico evaluado en TEDS-M. En J. L. González, J. A. Fernández-Plaza, E. Castro-Rodríguez, M. T. Sánchez-Compaña, C. Fernández, J. L. Lupiáñez y L. Puig (Eds.), Investigaciones en Pensamiento Numérico y Algebraico e Historia de las Matemáticas y Educación Matemática - 2014 (pp. 93-99). Málaga: Departamento de Didáctica de las Matemáticas, de las Ciencias Sociales y de las Ciencias Experimentales y SEIEM.

Hardy, I. (2014). A logic of appropriation: enacting national testing (NAPLAN) in Australia. Journal of education policy, 29(1), 1-18.

Hill, H. y Ball, D. (2004). Learning mathematics for teaching: Results from California's mathematics professional development institutes. Journal of Research in Mathematics Education, 35, 330-351.

INEE. (2012a). PIRLS - TIMSS 2011 Estudio Internacional de progreso en comprensión lectora, matemáticas y ciencias. Volumen I: Informe español. Madrid: Autor.

- (2012b). PIRLS - TIMSS 2011 Estudio Internacional de progreso en comprensión lectora, matemáticas y ciencias. Volumen I y II: Informe español. Madrid: Autor.

- (2012c). TEDS-M. Estudio internacional sobre la formación inicial en matemáticas de los maestros. Informe español. Madrid: Autor.

- (2012d). TEDS-M. Estudio internacional sobre la formación inicial en matemáticas de los maestros. Informe español. Análisis secundario. Madrid: Autor.

- (2013a). PISA 2012: Programa para la evaluación internacional de los alumnos. Informe español. Volumen I: Resultados y contexto. Madrid. Autor. 
- (2013b). PISA 2012: Programa para la evaluación internacional de los alumnos. Informe español. Volumen II: Análisis secundario. Madrid. Autor.

Martin, M.O. y Mullis, I.V.S. (2013). TIMSS AND PIRLS 2011: Relationships among reading, mathematics, and science achievement at the fourth grade - Implications for early learning. Martin, M.O. y Mullis, I.V.S. (Eds.). Chestnut Hill, MA: TIMSS \& PIRLS International Study Center, Boston College.

Ministerio de Educación y Ciencia (MEC) (1991). Real Decreto 1440/1991, de 30 de agosto, por el que se establece el título universitario oficial de Maestros en sus diversas especialidades y las directrices generales propias de los planes de estudios conducentes a su obtención. BOE, 244, 33004-33008.

- (2007a). Orden ECI/3857/2007, de 27 de diciembre, por la que se establecen los requisitos para la verificación de los títulos universitarios oficiales que habiliten para el ejercicio de la profesión de Maestro en Educación Primaria. BOE, 312, 53747-53750.

- (2007b). Orden ECI/2211/2007, de 12 de julio, por la que se establece el currículo y se regula la ordenación de la Educación primaria. BOE, 173, 31487-31566.

Ministerio de Educación, Cultura y Deporte (MECD) (2013a). PISA 2012. Programa para la evaluación internacional de los alumnos. Informe español. Volumen I: Resultados y contexto. Madrid: Autor.

- (2013b). Ley Orgánica 8/2013, de 9 de diciembre, para la mejora de la calidad educativa. BOE, 295, 97858-97921.

Montalvo, J. y Gorgels, S. (2013). Calidad del profesorado, calidad de la enseñanza y aprendizaje: resultados a partir del TEDS-M. En INEE (Ed.), TEDS-M. Estudio internacional sobre la formación inicial en matemáticas de los maestros. Informe español. Volumen II. Análisis secundario (pp. 11-39). Madrid: Autor.

Mullis, I. V. S., Martin, M. O., Ruddock, G. J., O’Sullivan, C. Y., Arora, A., y Erberber, E. (2007). TIMSS 2007 assessment frameworks. Chestnut Hill, MA: Boston College.

OCDE (2013). PISA 2012 Results in Focus. OECD Publishing.

Rico, L. (2013). El método del Análisis Didáctico. Unión, 33, 11-27.

Rico, L., Gómez, P. y Cañadas, M. C. (2014). Formación inicial en educación matemática de los maestros de primaria en España, 1991-2010. Revista de Educación, 363, 35-59.

Rico, L., Sierra, M. y Castro, E. (2002). El área de conocimiento de «Didáctica de la Matemática». Revista de Educación, 328,35-58. 
Schmidt, W. H., Blömeke, S., y Tatto, M. T. (Eds.). (2011). Teacher education matters: A study ofmiddle school mathematics teacher preparation in six countries. New York, NY: Teachers CollegePress.

Shulman, L. S. (1987). Knowledge and teaching: foundations of the new reform. Harvard Educational Review, 57(1), 1-22.

Tatto, M. T., Schwille, J., Senk, S., Ingvarson, L., Peck, R., y Rowley, G. (2008). Teacher Education and Development Study In Mathematics (TEDS-M): policy, practice, and readiness to teach primary and secondary mathematics. Conceptual framework. East Lansing, MI: Teacher Education and Development International Study Center, College of Education, Michigan State University.

Tatto, M. T., Schwille, J., Senk S., Ingvarson, L., Rowley, G., Peck, R., Bankov, K., Rodriguez, M., \& Reckase, M. (2012). Policy, Practice and Readiness to Teach Primary and Secondary Mathematics in 17 Countries: Findings from the IEA Teacher Education and Development Study in Mathematics (TEDS-M). Amsterdam: International Association for the Evaluation of Educational Achievement (IEA).

Villegas, J. L., Castro, E. y Gutiérrez, J. (2009). Representaciones en Resolución de Problemas: Un estudio de caso con problemas de optimización. Electronic Journal of Research in Educational Psychology, 7(1), 279-308. 


\section{Anexo}

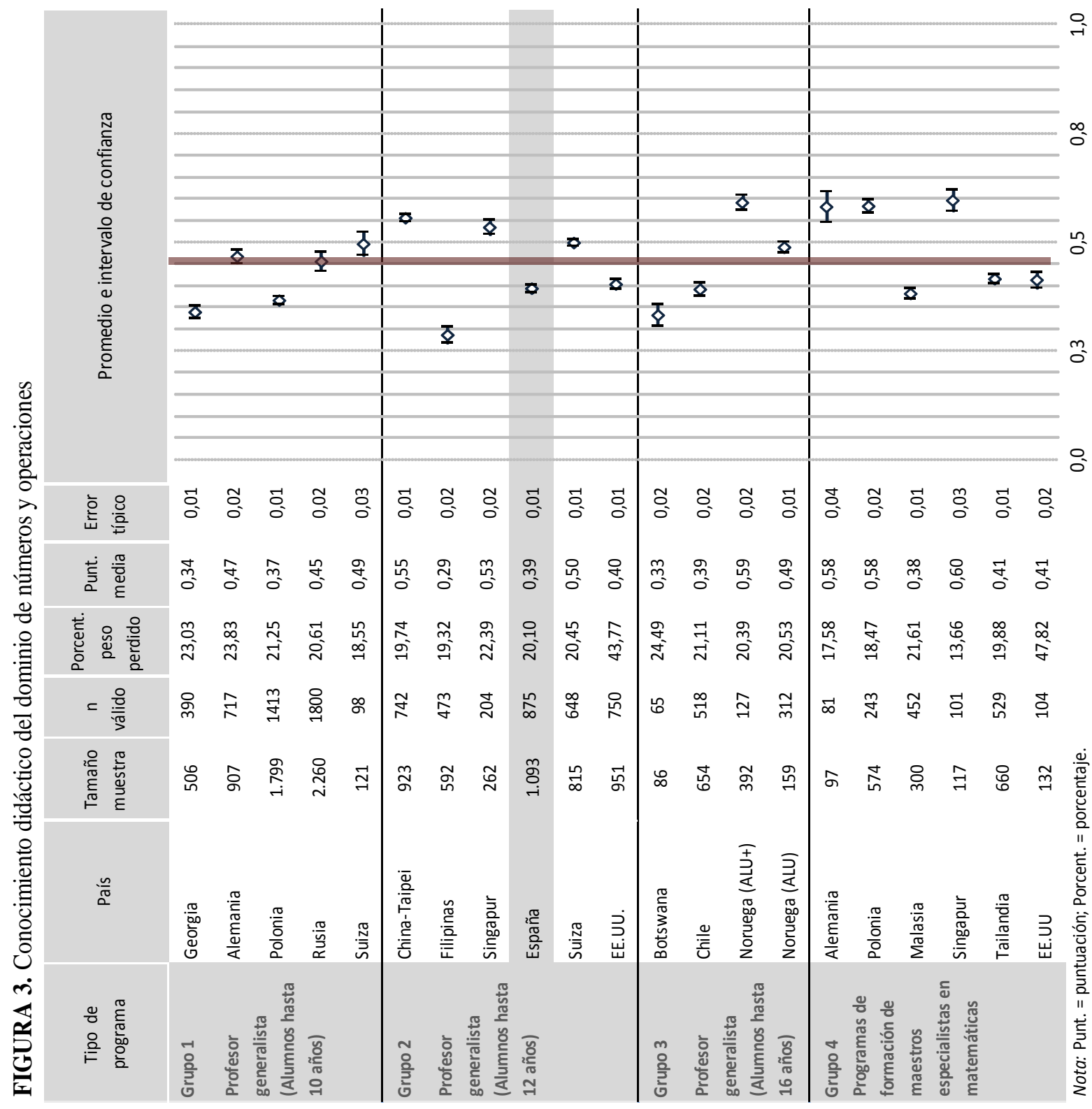




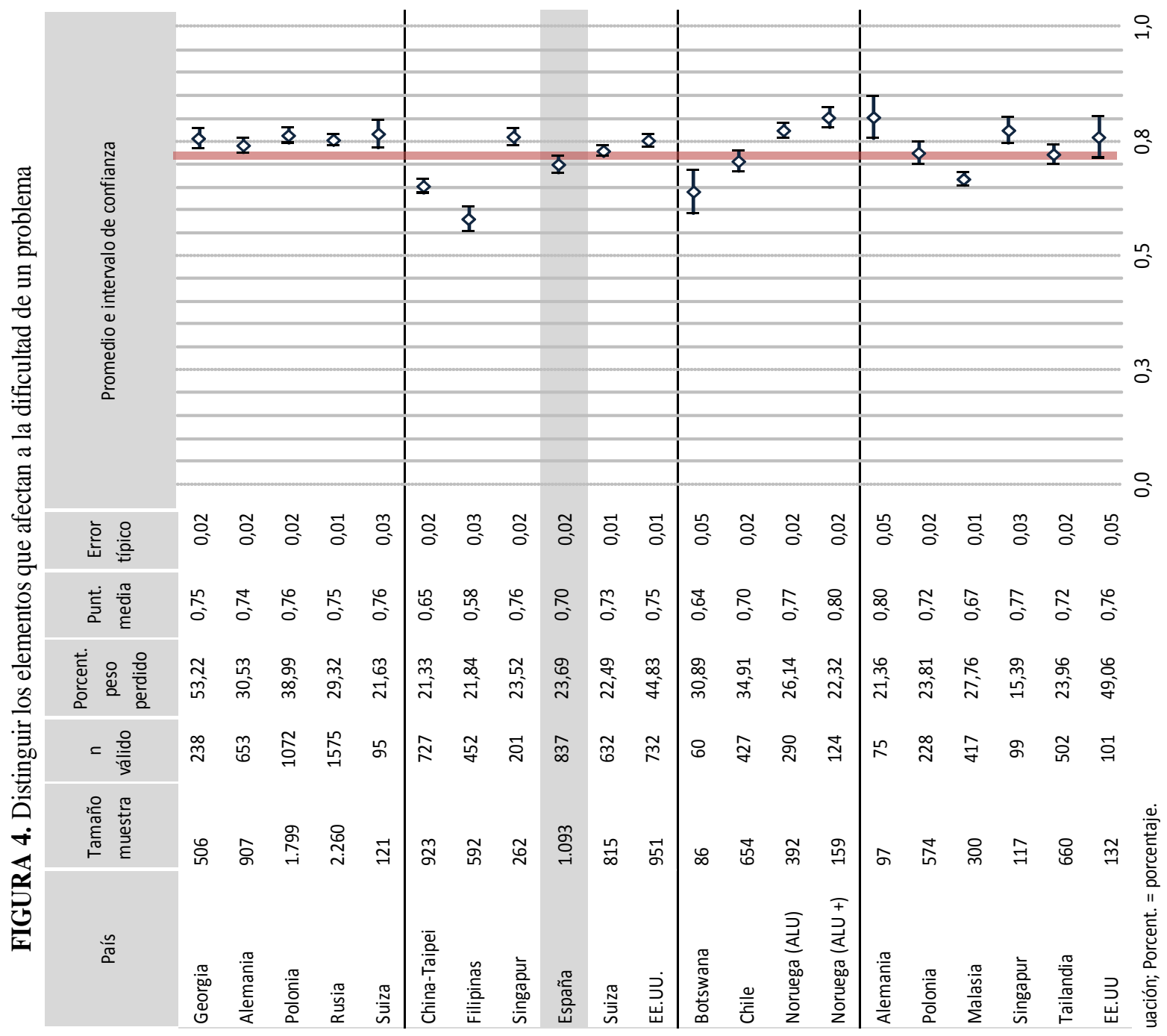




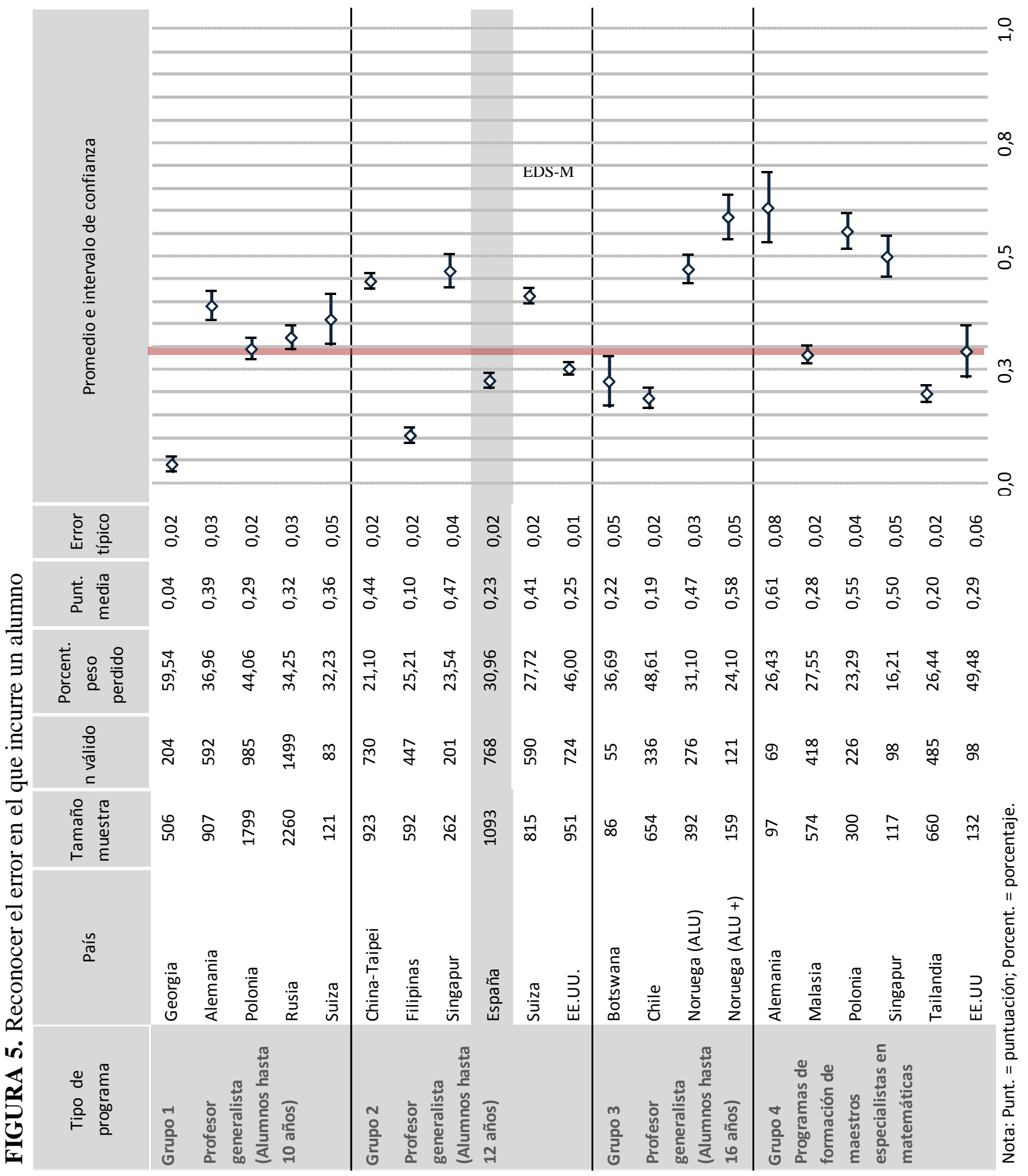

